\title{
Loss Aversion Underlying Violation
}

\author{
Atsuo Murata \\ Okayama University, Okayama, Japan
}

\begin{abstract}
In order to get insight into the prevention of violation-based human errors or accidents, loss aversion was discussed in detail. We paid attention to the way of earning money, and two ways of earning money were assumed in the experiment. The two ways of earning money included: (1) earn by oneself, (2) happen to get money by a lottery or in a prize. For these two cases, an attempt was made to evaluate the feelings of gain and loss using ME (magnitude estimation) method. On the basis of such an experiment, it was discussed how the extent of loss aversion differed according to the amount of value added to money (gain or loss). It was experimentally verified that we dislike losses more than we like commensurate gains. In other words, our feeling (regret) or attitude to the loss of some money $(\$ X)$ was larger than our feeling (pleasure) to the same gain of money $(\$ X)$. Moreover, such a difference was found to differ between situations under which gain or loss was induced. The difference of feeling between loss and gain was larger when the money was earned by oneself than when the money was earned by coincidence. When the money was incidentally got by a lottery or in a prize, the difference of feeling (value) between gain and loss was not so large as pointed out in prospect theory.
\end{abstract}

Keywords: loss aversion, prospect theory, loss, gain, ME (magnitude estimation), situation of loss or gain

\section{Introduction}

Recently, a lot of accidents which stem from violation (violating safety rule) frequently occur. These accidents include crucial accidents such as unintended crucial accidents like critical mass accident at the uranium processing plant of JCO Tokai Works Test Facility (Chiles, 2002), derailment accident in Fukuchiyama Line at JR West (West Japan Railway Company), Chernobyl nuclear power plant explosion, and NASA space shuttle explosion accident (Vaughan, 1997). In spite of increasing opportunity of accessing accident data base on the basis of the detailed accidental analysis, similar crucial accidents repeatedly occur.

Violation is committed through a prior intention to conduct this behavior (Reason, 2006). On the roads, violations are relatively easy to observe. Errors are committed without a prior intention. Error and violations seem to be mediated by different cognitive mechanisms. Murata and Nakamura (2014) discussed how cognitive biases distort decision-making and lead to crucial accidents using analytical examples of critical mass accident at the uranium processing plant of JCO Tokai Works Test Facility and NASA space shuttle explosion accident. It is impossible to separate accidents, in particular, from the psychological and cognitive characteristics of persons involved in the accident. Therefore, the understanding of psychological and cognitive behind the accident plays a more significant role for the prevention of violation than the data bases of crucial accidental analyses.

Atsuo Murata, Professor, Department of Intelligent Mechanical Systems, Graduate School of Natural Science and Technology, Okayama University. 
Loss aversion (Kahneman \& Tversky, 1979; 1984; 2000) is a cognitive property concerning the decision-making under risk. The fear of loss often produces an instinctual reaction against highly risky situations, and results in aversive behaviors of risky choices that can get higher gains. In general, our feeling (regret) to the loss of $\$ X$ is larger than our feeling (pleasure) the gain of $\$ X$. Such a property is strongly related to the violation-based human errors or accidents. When the management situation of a company gets worse, and a company is pressed for fund (money), the company feels the same amount of money more valuable as compared with the company whose management situation doesn't get worse and is not pressed for fund (money). In such a situation, the difference of the feeling between gain and loss is enlarged, and consequently the negative feeling to a major loss gets stronger. If the management situation of a company is getting worse and the company frantically attempts to compensate for the worse situation or deteriorated profit, such a negative feeling related to loss aversion must induce the urgency to get back the loss and lead to a trigger of violation behavior to compensate for the loss.

It is reasonable to think that the extent of loss aversive behavior differs according to the amount of relative value added to money (gain or loss). In other words, the intensity of loss aversive behavior measured by the difference of absolute value between loss and gain must be also affected by the situation under which such a loss or a gain is induced. However, there are few attempts to evaluate the extent of loss aversive behavior quantitatively according to the situation under which a loss or a gain is induced.

In order to get insight into the prevention of violation-based human errors or accidents, loss aversion was discussed in detail. We paid attention to the way of earning money, and two ways of earning money were assumed in the experiment. The two ways of earning money included: (1) earn by oneself; (2) happen to get money by a lottery or in a prize. We judged that the relative value added to money differed between two cases (1) and (2). For these two cases, an attempt was made to evaluate the psychological feelings of loss and gain using ME method. Here, it must be noted that the psychological feeling of loss and gain corresponds to the value function in prospect theory. On the basis of such an experiment, it was discussed how the extent of loss aversion differed according to the amount of relative value added to money (gain or loss).

\section{Loss Aversion}

The concept of loss aversion was proposed and theorized in prospect theory by Kahneman and Tversky (1979; 1984; 2000), Kahneman (2011) and Tversky and Kahneman (1974). It is apparent that we dislike losses more than we like commensurate gains. We are more upset when we loose something than when we get the same thing. We are more upset when we noticed that we had lost $\$ 100$ than we had obtained $\$ 100$. Loss aversion is reflected here. This is schematically shown in the value function in Figure 1. Therefore, loss aversion can be defined as a property to avoid loss by any means. It must be noted that it has not been explored how this property of loss aversion changes with the change of relative monetary value. Moreover, it has not been systematically shown how the value function changes for loss and gain domains. 


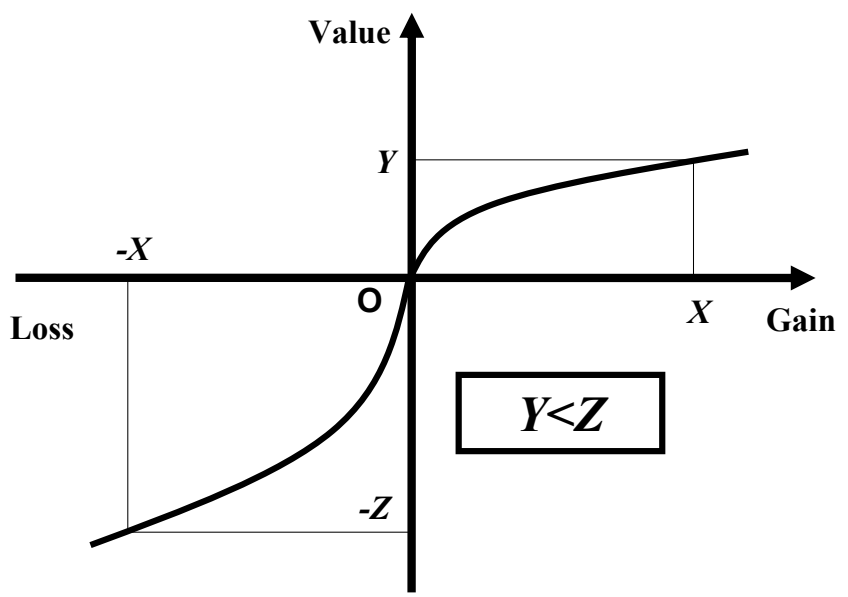

Figure 1. Value function and explanation of loss aversion.

\section{Method}

\section{Participants}

170 graduate or undergraduate students from 22 to 28 years old took part in the experiment. All of them majored in system engineering in undergraduate or graduated school. All participants agreed with the participation in this experiment after receiving a brief explanation on the experiment.

\section{Task}

In order to create feelings of gain and loss, the participant was required to conduct the following psychological evaluation. Five kinds of amount of money (10,000 yen, 25,000 yen, 50,000 yen, 75,000 yen, and 100,000 yen) were used in order to get the feeling of loss and gain for these amounts of money by making use of ME method (Stevens, 1957). Magnitude estimation method is a method to estimate the sensory magnitude induced by the stimulus by pre-specifying the sensory magnitude of one stimulus, and making a comparison with this standard stimulus. In other words, the relationship between the physical quantity and the corresponding sensory magnitude (psychological quantity) can be derived by this method. In this study, as the standard for the evaluation, the participants were given an instruction that the feeling of gain of 10000 yen was 100. The relationship between the physical quantity of the stimulus $S$ (amount of money) and psychological quantity $R$ (feeling of loss or gain) can be modeled according to the following equation.

$$
R=k S^{n}
$$

Here, $k$ and $n$ are empirically determined parameters. The participants were instructed not to use zero as a evaluation score of loss or gain, and evaluate their feeling to the amount of money using only a positive number for both loss and gain situations. The participants were also instructed that they can use any positive number as their evaluation score.

\section{Procedure}

This study assumed two ways of earning money: (1) earn by oneself; (2) happen to get money by a lottery or in a prize. This was a within-subject factor. The order of performance of two conditions was counterbalanced across the participants.

After giving a detailed explanation on the experiment, and confirming that the participants understand the 
evaluation procedure correctly, the experiment was begun. The geometric mean of all participants was calculated using the formula in Equation (2) below. The variables $n$ and $x_{i}$ stand for the number of participants $(=170)$ and evaluation score of loss or gain for the $i$-th participant, respectively. This was regarded as the representative of $R$ in Equation (1).

$$
R=\sqrt[n]{x_{1} x_{2} x_{3} \cdots \cdots x_{n}}
$$

Equation (2)

\section{Results}

For the sake of convenience of calculation, the following Equation (3) was used instead of Equation (1).

$$
R=k^{\prime}(S / 10000)^{n}
$$

For four combination of the way of earning money and loss or gain, the relationship between $S$ and $R$ as shown in Equation (3) was obtained. The contribution (square of correlation coefficient between $S$ and $R$ ) of fitting the data to the model in Equation (3) was more than 0.9 for all of four combinations. The contribution was as follows. Loss (earned by oneself): 0.985 , gain (earned by oneself): 0.990 , loss (earned by chance): 0.986 , gain (earned by chance): 0.995 . In this study, the participants were given a criteria (reference) (100 points for gain of $\backslash 10000$ when earned by oneself) for evaluation. In Figure 2, the relationship between money (physical quantity) and psychological quantity for loss and gain is plotte as a function of the way of earning money. Figure 3 corresponds to the logarithmic representation (both axes are $\log _{10} R$ or $\log _{10} S$ ) of Figure 2.

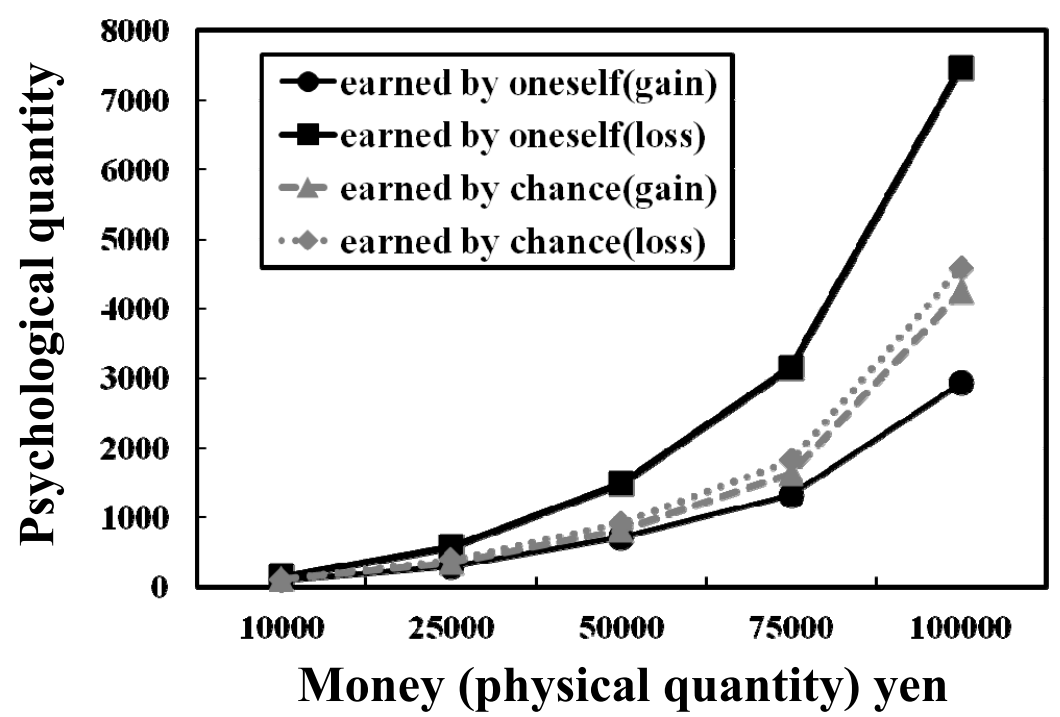

Figure 2. Relationship between money (physical quantity) and psychological quantity for loss and gain as a function of the way of loss or gain was induced.

As hypothesized in propsect theory, our feeling (regret) to the loss of $\$ X$ is larger than our feeling (pleasure) the gain of $\$ X$. It was also observed that the difference of psychological feeling between loss and gain was larger when earned by oneself than when earned by chance (see Figures 2 and 3). The estimated relationships between money (physical quantity) $S$ and psychological quantity (feeling or value of loss or gain) $R$ for the four cases (earned by oneself (gain), earned by oneself (loss), earned by chance (gain), and earned by chance (loss) in this order) are shown below. 


$$
\begin{gathered}
R=88.777(S / 10000)^{1.399} \\
R=131.129(S / 10000)^{1.636} \\
R=99.449(S / 10000)^{1.466} \\
R=94.471(S / 10000)^{1.548}
\end{gathered}
$$

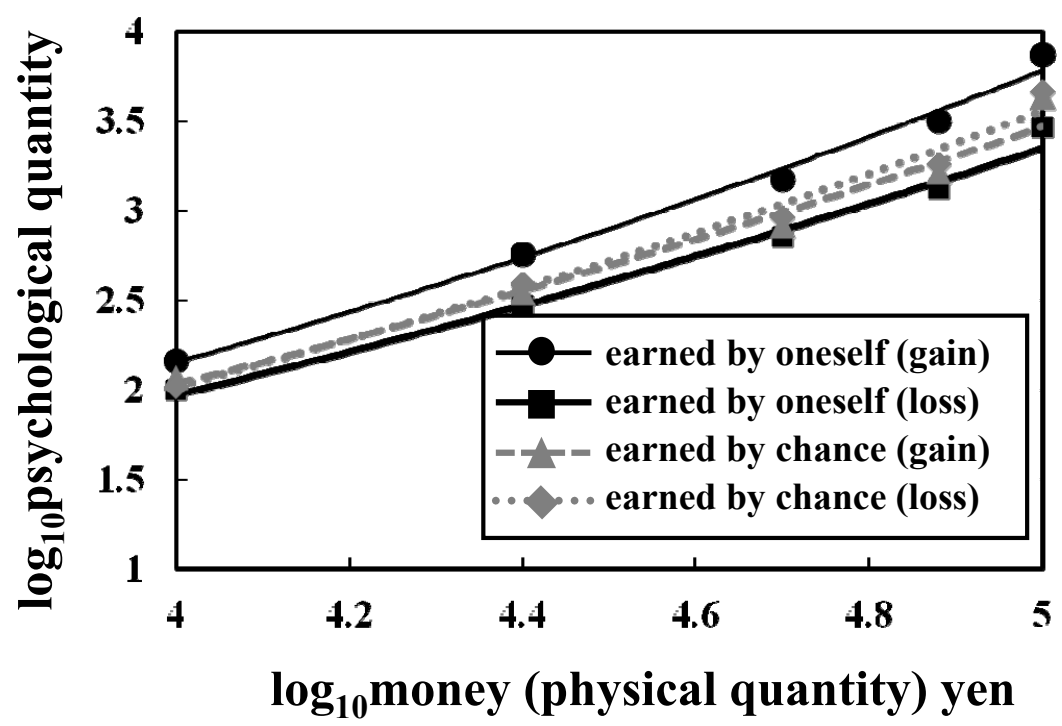

Figure 3. Logarithmic representation of relationship between money (physical quantity) and psychological quantity for loss and gain as a function of the way of loss or gain was induced.

\section{Discussion}

First, from Figures 2 and 3, it is clear that the relationship between the physical quantity (money) and the psychological quantity induced by loss or gain of the money can be represented by Setevens power law of Equation (1) (in this study, Equation (3)). As hypothesized, our feeling (regret) or attitude to the loss of some money was larger than our feeling (pleasure) to the same gain of the commensurate money. Moreover, such a difference of feeling between loss and gain was found to differ between situations under which gain or loss was induced. The difference of feeling between loss and gain got larger when the money was earned by oneself than when the money was earned by coincidence (see Figure 2).

In the case (1) (earn by oneself), the value of $R$ in the loss domain was by far larger than that in the gain domain (see Figure 2). On the other hand, in the case (2) (earn by chance), the value of $R$ did not differ so remarkably between the loss and the gain domain. It seems that the situation which is vulneerable and sensitive to loss aversion does not arise readily in such a case. Therefore, it is importtant to judge the situation on whether the loss aversion readily arises or not.

Kahneman and Tversky (1984) used the following value function to represent the relationship for both loss and gain domains. 


$$
v(x)=\left\{\begin{array}{l}
x^{\alpha} \\
-k(-x)^{\alpha}
\end{array}\right.
$$

Here, $\alpha$ is an empirically determined parameter, and $k$ is a positive constant. In prospect theory, the similar function is assumed for both loss and gain domains. The value function in the loss domain is different from that in the gain domain in that the coefficient $k$ is multiplied. As shown in Figure 2 and by Equations (5) and (6), the parameter $\alpha$ in Equation (8) should not be common to both loss and gain domains. In general, it has been shown that the parameter $k$ ranges from 2 to 2.5. However, the adjustment of value function $v(x)$ over all values of $x$ by the constant $k$ is not proper as shown in Figures 2 and 3. The difference of psychological quantity corresponding to the value function between loss and gain domains enlarged with the increase of money. The value function should be modeled separately for the loss and the gain domain, and a variety of explanation of phenomenon such as loss aversion should be reconsidered using the model described by Equations (5) and (6).

The tendency that difference of feeling between loss and gain got larger when the money was earned by oneself than when the money was earned by coincidence can be attributed to the endowment effect (Kahneman, 2011). The endowment effect worked so that the relative value of money earned by oneself was intensified. In such a situation where the relative value of money was enlarged, the corresponding loss of the same money must induce larger feeling (disappointment) of loss. The intensified relative value of money can be compared to the worse management situation of a company lacking in funds. The feeling of loss gets larger in such a situation than in an excellent management situation. Loss aversion is our property to avoid loss by all means due to the relatively stronger feeling to loss (fear or dislike) than the same gain. In other words, the situation of larger loss affects the emotion to avoid the loss right or wrong, and thus induced emotion becomes a trigger to the violation behavior to avoid larger loss. In such a way, it might be speculated that the larger the difference between the feelings of loss and gain, the more readily the violation behavior to avoid further loss is committed.

Future research should verify how such a loss aversive situation, that is, the larger difference of psychological feeling between loss and gain induces defective (non-cooperative) behavior corresponding to the violation behavior in repeated Prisoner's dilemma situation. Moreover, model development in prospect theory for explaining a variety of irrational phenomena such as loss aversion or framing effect should be reconsidered using the value function models in this study (Equation (3) and Equations (4)-(7)).

\section{Conclusion}

In order to get insight into the prevention of violation-based human errors or accidents, loss aversion was discussed in detail. We paid attention to the way of earning money, and two ways of earning money were assumed in the experiment. The two ways of earning money included: (1) earn by oneself; (2) happen to get money by a lottery or in a prize. For these two cases, an attempt was made to evaluate the feelings of gain and loss using ME method. On the basis of such an experiment, it was discussed how the extent of loss aversion differed according to the amount of value added to money (gain or loss).

As assumed, it was experimentally verified that we dislike losses more than we like commensurate gains. In other words, our feeling (regret) or attitude to the loss of some money $(\$ X)$ was larger than our feeling (pleasure) to the same gain of money $(\$ X)$. Moreover, such a difference was found to differ between situations under which gain or loss was induced. The difference of feeling between loss and gain was larger when the money was earned by oneself than when the money was earned by coincidence. 
Different from Kahneman and Tversky (1984) where the basically same value function is used for both loss and gain domains like Equation (8), it was suggested that the value function should be different for loss and gain domains as long as this study is concerned.

Future research should discuss how the finding on loss aversion should be effectively utilized for the prevention of violation-based crucial accidents. It must be explored how the degeneration of management (financial) situation of a company or the excessive pressure to the profit is related to the violation of social norms or rules on the basis of statistical data base of scandals or crucial accidents. The check mechanism of loss aversion and its vulnerability to crucial accidents or social scandals should be developed.

\section{References}

Chiles, J. R. (2002). Inviting disaster-Lessons from the edge of technology. Harper, N.Y.: New York.

Kahneman, D., \& Tversky, A. (1979). Prospect theory: An analysis of decision under risk. Econometrica, 47, $263-291$.

Kahneman, D., \& Tversky, A. (1984). Choices, values, and frames. American Psychologist, 39(4), 341-350.

Kahneman, D., \& Tversky, A. (2000). Choices, values, and frames. Cambridge University Press.

Kahneman, D. (2011). Thinking, fast and slow. Penguin Books.

Murata, A., \& Nakamura, T. (2014). Basic study on prevention of human error: How cognitive biases distort decision making and lead to crucial accidents. Proc. of AHFE, 2014, 136-141.

Reason, J. (2006). Human error. Cambridge University Press.

Stevens, S. S. (1957). On the psychophysical law. Psychological Review, 64(3), 153-181.

Tversky, A., \& Kahneman, D. (1974). Judgment under uncertainty: Heuristics and biases. Science, 185(4157), $1124-1131$.

Vaughan, D. (1997). The challenger launch decision-risky technology, culture, and deviance at NASA. The University of Chicago Press. 